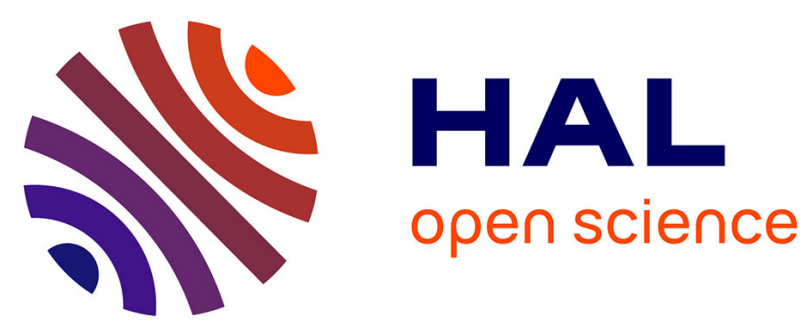

\title{
Premature gut and neonatal stress: high intestinal glucocorticoid responsiveness in early life shapes the risk of epithelial barrier defect in response to maternal separation
}

Nabila Moussaoui, Afifa Ait-Belgnaoui, Viorica Braniste, Eric Gaultier, Sandrine Ménard, Maïwenn Olier, Vassilia Theodorou, Pascal G.P. Martin, Eric Houdeau

\section{- To cite this version:}

Nabila Moussaoui, Afifa Ait-Belgnaoui, Viorica Braniste, Eric Gaultier, Sandrine Ménard, et al.. Premature gut and neonatal stress: high intestinal glucocorticoid responsiveness in early life shapes the risk of epithelial barrier defect in response to maternal separation. Digestive Disease Week (DDW), May 2014, Chicago, United States. hal-01603423

\section{HAL Id: hal-01603423 https://hal.science/hal-01603423}

Submitted on 5 Jun 2020

HAL is a multi-disciplinary open access archive for the deposit and dissemination of scientific research documents, whether they are published or not. The documents may come from teaching and research institutions in France or abroad, or from public or private research centers.
L'archive ouverte pluridisciplinaire HAL, est destinée au dépôt et à la diffusion de documents scientifiques de niveau recherche, publiés ou non, émanant des établissements d'enseignement et de recherche français ou étrangers, des laboratoires publics ou privés. 
and plasma GLP-1 in overweight and obese adults with genetic variation at loci controlling pathways of interest: adrenergic (ADR2A, ADRB3); fat mass (FTO), receptor-cellular function (GNB3), serotonergic (5-HTTLPR), GLP-1 pathway (TCF7L2), thermogenesis (UCP-2, UCP3), bile acids (GPBAR1) loci. Methods: 271 overweight or obese, predominantly Caucasian, participants underwent, on separate days, validated measurements of intermediate phenotypes: GE of solids and liquids by scintigraphy, gastric volume (GV) by SPECT during fasting and after $200 \mathrm{~mL}$ Ensure ${ }^{\circledR}(\Delta \mathrm{GV})$, satiety by kcal intake (T-kcal) during a buffet meal $4 \mathrm{~h}$ after the Ensure ${ }^{\circledR}$ meal, satiation (maximal tolerated volume, MTV) by Ensure ${ }^{\circledR}$ ingested at $30 \mathrm{~mL} / \mathrm{min}$, and fasting and postprandial GLP-1 (by radioimmunoassay). We genotyped, with TaqMan ${ }^{\circledR}$ SNP Assay, 16 variants in the genes of ADR2A, ADRB3, UCP-2, UCP-3, FTO, 5-HTTLPR, TCF7L2, GNB3, and GPBAR1 [minor allele frequencies (MAF) in table]. We assessed the association of each genotype with each intermediate phenotype using an ANCOVA (adjusting for age and gender). Results: 271 participants (188 females, 83 males), mean age $36.7 \pm 1.2 \mathrm{y}$, BMI $32.9 \pm 0.3 \mathrm{~kg} / \mathrm{m}^{2}$. The table shows significant univariate associations of gene SNPs with the intermediate phenotypes (bolded for those with $\mathrm{p}<0.05$ ). The main findings are summarized by mechanism or pathway: adrenergic, ADRA2C (rs2234888) with GE of liquids; fat mass, FTO (rs9939609) with GE of solids and liquids; GLP-1 pathway, TCF7L2 (rs7903146) with GE of solids; thermogenesis UCP3 (rs2075577) with satiation, (rs15763) with satiety, and (rs1626521) with GE of liquids, $\Delta G V$, satiation and satiety; and bile acids, GPBAR1 (rs11554825) with satiation. Conclusion: Genes associated with T2DM or obesity also alter certain gastric motor functions, satiation or satiety. Variants in UCPS are associated with all three domains of energy intake investigated, that is, gastric motor function, satiation and satiety. The FTO (A) allele is associated with accelerated GE for solids and liquids, suggesting a pathophysiological mechanism for its association with development of obesity. The UCP data suggest that mitochondrial proteins alter gastric motor function in addition to known effects on energy metabolism, and both may impact food intake. Support: NIH DK67071

Univariate associations of obesity candidate genes with intermediate phenotypes

\begin{tabular}{|c|c|c|c|c|c|c|c|c|c|}
\hline $\begin{array}{c}\text { Gene } \\
\text { name }\end{array}$ & SNP & $\begin{array}{c}\text { MAF } \\
\text { among } \\
\text { locals }\end{array}$ & $\begin{array}{c}\text { LGE } \\
\text { T1/2 }\end{array}$ & $\begin{array}{c}\text { SGE } \\
\text { T1/2 }\end{array}$ & $\begin{array}{c}\text { SGE } \\
\text { lag }\end{array}$ & $\begin{array}{c}\Delta \\
\text { GV }\end{array}$ & MTV & VAS-agg & $\begin{array}{c}\text { Buffet } \\
\text { T-Kcal }\end{array}$ \\
\hline ADRA2A & $\mathrm{rs} 1800544$ & 0.295 & & & & & 0.10 & & 0.096 \\
\hline ADRA2C & $\mathrm{rs} 2234888$ & 0.05 & 0.035 & & & 0.074 & & & \\
\hline FTO & $\mathrm{rs} 9939609$ & 0.44 & 0.006 & 0.015 & & & & & \\
\hline GNB3 & $\mathrm{rs} 5443$ & 0.30 & & & 0.089 & & & & \\
\hline GNB3 & $\mathrm{rs} 1129649$ & 0.336 & & & & & & & \\
\hline GNB3 & $\mathrm{rs} 1047776$ & 0.073 & & 0.15 & & & 0.049 & & \\
\hline $5-H T T L P R$ & $\mathrm{rs} 4795541$ & 0.41 & & 0.15 & 0.12 & & & & \\
\hline TCF7L2 & $\mathrm{rs} 7903146$ & 0.22 & & 0.09 & 0.047 & & & & \\
\hline UCP2 & $\mathrm{rs} 659366$ & 0.372 & & & 0.079 & & 0.11 & & \\
\hline UCP2 & -3474, & 0.24 & & & & & & 0.11 & 0.064 \\
\hline UCP3 & $\mathrm{rs} 2075577$ & 0.404 & & 0.15 & & & 0.036 & & 0.15 \\
\hline UCP3 & $\mathrm{rs} 15763$ & 0.25 & 0.15 & & & & 0.087 & & 0.03 \\
\hline UCP3 & $\mathrm{rs} 1626521$ & 0.267 & 0.02 & & & 0.005 & 0.045 & 0.13 & 0.005 \\
\hline GPBAR1 & $\mathrm{rs} 11554825$ & 0.41 & & 0.059 & 0.12 & & & 0.048 & 0.15 \\
\hline
\end{tabular}

$\mathrm{MAF}=$ minor allele frequency; $\mathrm{GE}=$ gastric emptying; $\mathrm{LGE}=\mathrm{GE}$ of liquids; $\mathrm{SGE}=\mathrm{GE}$ of solids; $\Delta \mathrm{GV}=$ gastric accommodation and volume; MTV=Maximal tolerated volume; VAS-agg=visual analog score -aggregate of 4 symptoms (nausea, bloating, fullness, pain); SNP=single nucleotide polymorphisms; T-Kcal=total kcal

\section{7}

The Role of Toll-Like Receptor-4 in Gut-Brain Cross Talk in a Murine Model of Parkinson's Disease

Paula Perez-Pardo, Hidde Douna, Tom Wijnands, Sofia Lopes da Silva, Christopher B. Forsyth, Hemraj B. Dodiya, Johan Garssen, Berend Olivier, Ali Keshavarzian, Aletta D. Kraneveld

Background. Gastrointestinal dysfunction is a common symptom in patients suffering from Parkinson's disease (PD). The intestinal symptoms precede the onset of classical motor symptoms and $\alpha$-synuclein brain pathology by many years, and their occurrence in otherwise healthy people is associated with an increased risk of developing the disease. The intestine might be an early site reflecting the development of PD. Recently it was found that abnormal intestinal permeability correlated with $\alpha$-synuclein positive Lewy bodies in the enteric nervous system (ENS). Toll like receptors (TLRs) are expressed by innate immune cells in gut and TLR4 is required for $\alpha$-synuclein-induced proinflammatory activity of microglial cells in the brain. We hypothesize that intestinal TLR4 dysregulation may play a role in intestinal pathology of PD. Methods. Rotenone is a pesticide that inhibits Complex I of the mitochondrial respiratory chain. Rotenone exposure in rodents is a frequently used model for studying PD since it is able to reproduce PD pathological features found in the brain of PD patients. Male C57BL6J mice, TLR4 deficient and wild type mice were given rotenone orally ( $10 \mathrm{mg} / \mathrm{kg}$ daily by oral gavage for 28 days) and animals were sacrificed after 28 days. Control mice received vehicle. Rotarod tests were performed on days 5, 10, 15, 21 and 28 Intestinal transit time was assessed 28 days after surgery $(\mathrm{N}=10)$. Results. Rotenone induces motor deficits (disturbed rotarod handling), delayed intestinal transit time and $\alpha$-synuclein accumulation in the ENS in mice. Moreover, we observed inflammation in the gut in rotenone-treated mice characterized by reduced colon length, an increased number of CD3 positive T cells and neutrophil infiltration. TLR4 deficient mice were partly protected from the development of rotenone-induced motor deficits and delayed intestinal transit time. No effect was observed on colon length reduction after rotenone treatment when comparing
TL4 deficient and wild type mice. Conclusions. Oral rotenone caused: 1. PD like motor deficits; $2 . \alpha$-synuclein pathology in ENS and 3. intestinal motility dysfunction and inflammation. TLR4 seems partly involved in the motor dysfunction and intestinal phenotype found after rotenone administration. Our results support the hypothesis that gut/brain cross talk possibly via TLR4 plays a central role in $\alpha$-synuclein-induced PD pathology.

\section{8}

Excessive Expression of TLR4 in Human Fetal Colonic Epithelial Cells Is Developmentally Regulated: A Possible Mechanism for Necrotizing Enterocolitis

Weishu Zhu, Hai Ning Shi, Allan Walker, Di Meng, Lei Lu

Necrotizing enterocolitis (NEC) is a devastating neonatal intestinal inflammatory disease, occurring primarily in premature infants due to developmental immaturity of intestinal host defense. Evidence has indicated a critical role for the receptor for bacterial lipopolysaccharide (LPS) toll like receptor 4 (TLR4) in the pathogenesis of NEC. But the expression pattern of TLR4 in immature intestine and its regulatory mechanism is unclear. Amniotic fluid and breast milk contain trophic factors such as hydrocortisone (HC) and TGF beta that interac with the gut to stimulate the development of host defenses and can attenuate the severity of NEC. The objective of this study was to investigate gestational age and HC-induced changes in TLR4 (cell surface VS subcellular) on human fetal colonic epithelium (all experiments involving utilizing human tissues were approved by Partners for Human Research Committee. Protocol \# 1999-P- 003833). TLR4 localization in human fetal colonic epithelium at different gestational ages (10-21 weeks) and with or without HC stimulation was assessed using immunofluorescence confocal microscopy. The expression of TLR4 was detected on the apical surface, and in the cytoplasm and basal surface in human fetal colonic epithelium from 10 - 21 weeks in 18 samples. The surface to cytoplasm ratio of TLR4 fluorescence intensity was higher from 10 to 16 weeks gestational age, and was reduced from 20 to 21 weeks gestational age. The reduction of surface to cytoplasm ratio of TLR4 fluorescence intensity was also observed after 20-hour of $\mathrm{HC}$ stimulation in 3 fresh colonic tissues at 16 weeks gestational age. The similar results were observed in fetal human colonic epithelial cell line (FHC, from ATCC). In this cell line the surface to cytoplasm ratio of TLR4 fluorescence intensity was reduced after 5 days of $1 \mu \mathrm{M}$ HC stimulation compared with control group At the same time HC treatment inhibited LPS induced IL-6 induction, which was observed in control group, indicating that HC changing TLR4 distribution might affect TLR4 signaling in the FHC cell line. In summary, these results suggest that location and intensity of TLR4 expression in fetal colonic epithelial cells might be regulated by gestational age and established trophic factors such as HC, which in turn affects TLR4 signaling. These observations may be crucial to our understanding of the mechanism of TLR4 movement in NEC.

249

Premature Gut and Neonatal Stress: High Intestinal Glucocorticoid Responsiveness in Early Life Shapes the Risk of Epithelial Barrier Defect in Response to Maternal Separation

Nabila Moussaoui, Afifa Ait-Belgnaoui, Viorica Braniste, Eric Gaultier, Sandrine Ménard, Maïwenn Olier, Vassilia Theodorou, Pascal G. Martin, Eric Houdeau

Background: Glucocorticoids (GCs) contribute to human intestine ontogeny and accelerate gut barrier development in preparation to birth. In comparison, the rat gut is immature at birth, and high intestinal GCs sensitivity during the first two weeks of life resembles that of premature infants. This makes suckling rats a model to investigate postpartum impact of maternal separation (MS)-associated GC release in preterm babies. Our aim was to determine the effects of an episode of MS on epithelial barrier integrity, and whether high intestinal GC sensitivity at birth may shape MS effects in an immature gut. Methods: Randomized rat pups at postnatal day (PND) 10 or PND20 were separated from their dams for $4 \mathrm{~h}$ (MS), or handled but left undisturbed with their dams during the $4 \mathrm{~h}$ period (sham). Total in vivo intestinal permeability (IP) was assessed through mucosal-to-blood passage of FITC-Dextran $4 \mathrm{kDa}$ (FD4) orally administered to pups with or without ML7, an inhibitor of myosin lightchain (MLC) kinase (lmg/kg BW i.p.), or the glucocorticoid receptor (GR) antagonist RU486 $(2 \mathrm{mg} / \mathrm{kg}$ BW s.c.). By Ussing chambers, colonic and ileal permeability to FD4 and to horseradish peroxidase (HRP 44kDa) were measured at PND10 and PND20 with or without MS. Bacterial translocation (BT) was assessed in liver and spleen 24h after MS or sham procedure. Additional groups of PND10 and PND20 pups were used for comparative qPCR analysis of GR expression in the colon and the ileum, and dose-effect of Dexamethasone (DEX) on total IP to FD4. Results: A 4h-MS applied once at postnatal day (PND)10 enhanced plasma corticosterone in male and female pups, increased total IP to FD4 immediately after the end of MS (2-fold; $\mathrm{p}<0.01)$, and induced BT to liver and spleen compared to sham pups. Ussing chamber experiments demonstrated a 2 -fold increase of permeability to FD4 in the colon of MS pups $(\mathrm{p}<0.01)$, but not in the ileum. Colonic permeability was not only increased for FD4 after MS but also to intact HRP 44kDa demonstrating MS-induced transepithelial passage of high molecular weight macromolecules. In vivo, the GR antagonist RU486 or ML7 blockade of MLC-kinase controlling epithelial cytoskeleton contraction prevented MS-induced IP increase to FD4 and BT, while the GR agonist DEX dose-dependently mimicked MS-increase of IP to FD4 (ED50 $0.1 \mathrm{mg} / \mathrm{kg}$ ). In contrast, MS effects on IP and BT were absent at PND20, a model of full-term infant, characterized by a marked drop in IP response to DEX (i.e. no response before $1 \mathrm{mg} / \mathrm{kg}$ ), and decreased GR expression in the colon only compared to PND10 pups. Conclusion: These results show that high intestinal GC responsiveness in a rat model of prematurity defines a vulnerable window for a postdelivery MS, evoking immediate disruption of epithelial integrity in the large intestine, and increasing susceptibility to macromolecule passage and bacteremia. 